\title{
Preferences and aversions for stimuli paired with ethanol in hungry rats
}

\author{
J. E. SHERMAN, C. F. HICKIS, A. G. RICE, K. W. RUSINIAK, and J. GARCIA \\ University of California, Los Angeles, California
}

\begin{abstract}
Two experiments explored the reinforcing effect of ethanol on conditioned location and flavor preferences in hungry rats. In Experiment 1, rats were administered ethanol (.5, 1.0, or $2.0 \mathrm{~g} / \mathrm{kg}$, ig) prior to confinement in one side of a shuttlebox with access to a flavored solution. On control trials, $\mathrm{H}_{2} \mathrm{O}$ was administered prior to confinement to the opposite side with a different flavored solution. Location choice tests revealed an overall aversion for the ethanol-associated side that was largest at the $2.0-\mathrm{g} / \mathrm{kg}$ dose. Flavor choice tests revealed an aversion for the ethanol-associated flavor at the $2.0-\mathrm{g} / \mathrm{kg}$ dose, no reliable difference at the $1.0 \cdot \mathrm{g} / \mathrm{kg}$ dose, and, of particular interest, a preference at the .5-g/kg dose. The results of Experiment 2 suggest that caloric restoration served as the reinforcing mechanism for the conditioned flavor preference. An isocaloric glucose solution conditioned a flavor preference of the same magnitude as that obtained with ethanol. Moreover, when ethanol provided no caloric advantage, the associated flavor was less preferred than a flavor associated with an isocaloric glucose solution.
\end{abstract}

Animal models of alcoholism suffer from the drawback that it is difficult to show conditioned preferences for stimuli associated with ethanol. In fact, pairing ethanol with neutral stimuli typically produces a conditioned aversion. Such aversions have been demonstrated in the rat for both flavor cues (Cappell, LeBlanc, \& Endrenyi, 1973; Cunningham, 1979; Eckardt, Skurdal, \& Brown, 1974; Lester, Nachman, \& LeMagnen, 1970) and location cues (Cunningham, 1979, 1981). The fact that rats develop aversions to flavors paired with ethanol is difficult to reconcile with the fact that humans acquire preferences for flavors associated with the drug (e.g., the flavor of beer or Scotch). Moreover, while animal research shows that other drugs of abuse, such as morphine and amphetamine, will also produce conditioned flavor aversions, these drugs can be shown to yield conditioned preferences for location cues (Beach, 1957; Reicher \& Holman, 1977; Rossi \& Reid, 1976; Sherman, Pickman, Rice, Liebeskind, \& Holman, 1980; Sherman, Roberts, Roskam, \& Holman, 1980). Thus, in this context, the effects of ethanol observed in rats are inconsistent both with the apparent development of preferences for flavors paired with alcohol in humans and research with rats showing that other classes

This research was supported by National Institutes of Health Research Grant USPHS NS 11618 and Program Grants HD 05958 and AA 03513. Reprint requests should be addressed to the first author, Department of Psychology, UCLA, Los Angeles, California 90024. C. F. Hickis is now at the Chemical Dependency Center, West Park Country Hospital, Cody, Wyoming, and K. W. Rusiniak is at Eastern Michigan University. of drugs abused by humans will condition a location preference.

Recently, Stewart and Grupp (1981) found that after hungry rats were given ethanol and food in one location and only food in another location, a choice test revealed a conditioned preference for the ethanol-paired location; however, without food in training ethanol did not condition a preference. These findings suggest that ethanol may interact with food and/or hunger in ways that cannot be predicted from the effect of the drug when presented alone. This result is of interest because alcohol use in humans frequently occurs along with food consumption. It may be that the motivational state of hunger and/or the presence of food may be critical in yielding both ethanol-conditioned flavor preferences, as would be expected on the basis of human ethanol consumption, and conditioned location preferences, a result obtained with other commonly abused substances.

The present study explored this possibility employing the procedure used by Holman and his associates (Reicher \& Holman, 1977; Sherman et al., $1980 \mathrm{a}, 1980 \mathrm{~b}$ ) to study simultaneously the conditioned flavor and location effects of morphine and amphetamine. With this procedure, on some days rats are placed in one distinctive compartment of a two-compartment shuttlebox with a distinctive flavor present. On other days, the rats are placed in the other compartment with a different flavor present. One side of the shuttlebox and one flavor are consistently paired with the drug, the other side and flavor with the vehicle control. In this paradigm, rats are food deprived and food is presented as part of the flavor cue; that is, a $5 \%$ sucrose solu- 
tion is a common component of the distinctive flavors. Following training, location and flavor preference tests are conducted separately.

In Experiment 1 of the present study, these procedures were used to study three doses of ethanol: $.5,1.0$, and $2.0 \mathrm{~g} / \mathrm{kg}$. In Experiment 2, the contribution of caloric restoration to the conditioned effects observed in Experiment 1 was assessed.

\section{EXPERIMENT 1}

\section{Method}

Subjects. The subjects were 36 male Sprague-Dawley rats obtained from Simonsen Laboratories in Gilroy, California. The rats were 90-120 days old at the start of the experiment and were maintained at $85 \%$ of their free-feeding weights. All rats were individually housed and had free access to water in their cages. All procedures were conducted at least 2 weeks after the rats arrived at our vivarium and only during the light component of the 12-h light-dark cycle.

Apparatus and Drugs. Each rat was trained in one of three identical rectangular Plexiglas shuttleboxes $(47.5 \times 22.5 \times 28.5 \mathrm{~cm})$. Each box had a stainless steel grid floor and a removable Plexiglas barrier across the middle that restricted rats to the appropriate side of the shuttlebox during training sessions. Light gray cardboard behind the transparent Plexiglas walls and Plexiglas barrier faced the interior of the right side of each shuttlebox. Vertical stripes of 2-cm-wide black tape, $2 \mathrm{~cm}$ apart, were on the white cardboard facing the interior of the left side of each shuttlebox and on the left side of the otherwise transparent Plexiglas ceilings. Vertical strips of transparent, .7-cm-thick and 1.7-cm-wide Plexiglas were superimposed on the interior of the left side of each shuttlebox between the black tape (i.e., over the white vertical stripes). Thus, the sides of each box differed both visually (gray vs. black and white vertical stripes) and tactually (smooth vs. vertical ridges). During testing, the barrier used during training was replaced with an otherwise identical barrier with a rectangular $(25 \times 28 \mathrm{~cm})$ opening centered on the bottom that provided access to both sides of the shuttlebox. Also, the floor was free to tilt to the left or right, activating a microswitch that automatically signaled which side the rat occupied. The three shuttleboxes were located in a dimly illuminated experimental room with masking white noise $(62 \mathrm{~dB})$ present.

There were two flavored drinking solutions: the $\mathrm{HCl}$ solution contained $.1 \% \mathrm{HCl}$ and $5 \%$ sucrose, and the $\mathrm{NaCl}$ solution contained $3 \% \mathrm{NaCl}$ and $5 \%$ sucrose (all solutions were mixed by weight in tap water). These solutions were presented to the rats in graduated test tubes fitted with metal drinking spouts. The spouts projected through a small hole on each end of the shuttleboxes, $3.5 \mathrm{~cm}$ above the grid floor, thereby permitting access to the flavored solutions during training.

The rats were gastrically intubated (ig) either with distilled water alone or with distilled water mixed with ethanol $(95 \%)$ diluted to yield $.5,1.0$, or $2.0 \mathrm{~g} / 10 \mathrm{ml}$. The volume intubated was $10 \mathrm{ml} / \mathrm{kg}$. Thus, the resulting doses of ethanol were $.5,1.0$, and $2.0 \mathrm{~g} / \mathrm{kg}$, respectively.

Procedure. Prior to their first training day, all rats were sham intubated and given $20 \mathrm{ml}$ of $5 \%$ sucrose in their home cages to habituate them to the intubation procedure and reduce possible neophobic reactions to the flavored solutions presented during training. The drug schedule during a single training cycle consisted of six presentations of water and six of ethanol: water on Mondays and Thursdays and ethanol on Tuesdays and Fridays. On the other days of the week, the rats remained in their home cages. Thus, each cycle was concluded in 3 weeks. The rats were tested on the Sunday following the last presentation of ethanol. This cycle of training and testing was repeated three times.

On training days, all rats were weighed and intubated in the vivarium and 2-3 min later transported to the nearby experimental room, where they were exposed to a distinctive set of flavor and location cues uniquely associated with either water or ethanol. On water days, each rat was placed in one side of the shuttlebox for $20 \mathrm{~min}$ with access to $30 \mathrm{ml}$ of either the $\mathrm{HCl}$ - or $\mathrm{NaCl}$-flavored solution. On ethanol days, each rat was placed in the opposite side of the shuttlebox for $20 \mathrm{~min}$ with access to the other solution. Rats were fed a restricted amount of Purina Lab Chow 120-150 min after each training session to maintain them at $85 \%$ of their free-feeding weights.

The rats were assigned randomly to one of three groups $(\mathrm{n}=$ 12), each receiving a different dose of ethanol $(.5,1.0$, and $2.0 \mathrm{~g} / \mathrm{kg}$ ) during training. Half the rats in each group were placed on the left side of the shuttlebox on ethanol days and half on the right side. Additionally, half the rats in each group received the $\mathrm{HCl}$-flavored solution on the ethanol days and half received the $\mathrm{NaCl}$ solution.

On the test days, acquired location and flavor preferences were assessed. First, location preference was assessed. Each rat was placed in the black and white striped (and vertically ridged) side of the shuttlebox with the barrier that permitted access to either side. No solutions were present; the rat's location was automatically recorded for $\mathbf{2 0} \mathrm{min}$. Immediately after the location preference test, each rat was returned to its home cage and presented $30 \mathrm{ml}$ each of the $\mathrm{HCl}$ - and $\mathrm{NaCl}$-flavored solutions in adjacent test tubes for $20 \mathrm{~min}$. The position of the tubes was balanced across rats. Total consumption of each solution was recorded.

Statistical analyses. All statistical tests were conducted with the analysis of variance (ANOVA). For flavor, the dependent variable was amount consumed; the within-subject factor was flavor $(\mathrm{HCl}$ vs. $\mathrm{NaCl})$; the between-subject factor was reinforced cue (ethanol with $\mathrm{HCl}$ vs. ethanol with $\mathrm{NaCl}$ ). For location, the dependent variable was time spent on the left side; the betweensubject factor was reinforced cue (ethanol with left side vs. ethanol with right side). The rejection criterion for statistical significance was $\mathrm{p}<.05$.

\section{Results}

Figure 1 presents the mean consumption of the reinforced (ethanol-paired) and nonreinforced (vehicle-paired) flavored solutions for all three groups on each day of training and testing. As the figure suggests, there was a significant interaction between dose of ethanol and amount of consumption of the reinforced and nonreinforced solutions during training $[F(2,30)=19.2]$. Rats intubated with $.5 \mathrm{~g} / \mathrm{kg}$ of ethanol (top panel) consumed more of the ethanol-paired solutions $[\mathrm{F}(1,10)=21.5]$, whereas rats intubated with $2.0 \mathrm{~g} / \mathrm{kg}$ (bottom panel) drank less of the ethanol-paired solutions $[F(1,10)=25.6]$. Rats receiving the intermediate dose of ethanol, $1.0 \mathrm{~g} / \mathrm{kg}$ (middle panel), consumed slightly more of the ethanol-paired solutions, but this difference was not statistically significant $(F<1)$.

Because amount of each solution consumed during training was measured on different days and because consumption during training also reflects the possible contribution of unconditioned effects of ethanol, the two-choice preference test provides clearer evidence of conditioned effects. The test 


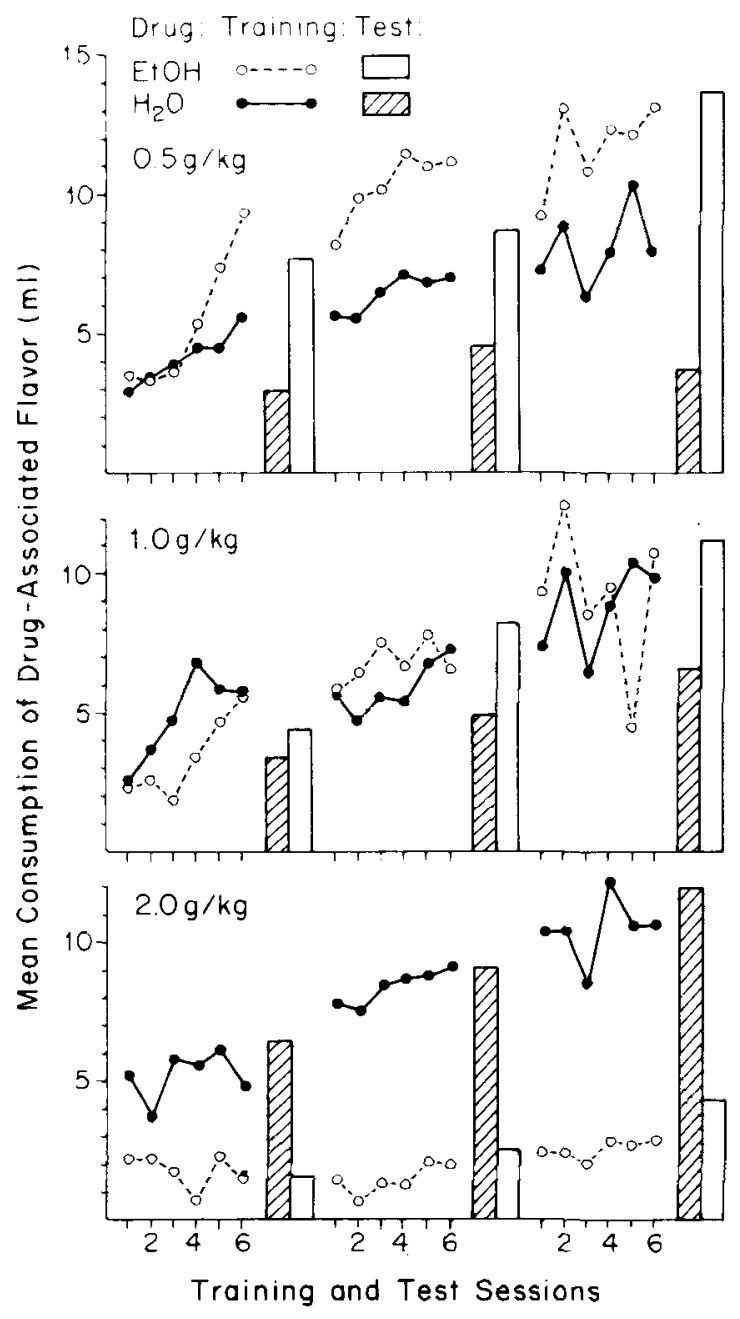

Flgure 1. Mean consumption of flavors associated with ethanol (ETOH) or water $(\mathrm{H}, \mathrm{O})$ during training and test sessions.

data, also shown in Figure 1, were highly consistent with the training results. An overall ANOVA of the consumption test data yielded a statistically significant interaction between dose and reinforced solution $[F(2,30)=16.5]$. Independent ANOVAs conducted for each group across all three tests show that the $.5-\mathrm{g} / \mathrm{kg}$ group displayed a significant preference for the ethanol-paired solutions $[F(1,10)=$ $72.0]$, the $1.0-\mathrm{g} / \mathrm{kg}$ group showed no significant difference in consumption between the ethanol- and vehicle-paired solutions $(F<1)$, and the $2.0-\mathrm{g} / \mathrm{kg}$ group displayed a significant aversion for the ethanolpaired solutions $[F(1,10)=13.4]$.

Table 1 presents the mean percentage of time spent on the side of the shuttlebox paired with ethanol for each of the three location preference tests for each group of rats. Overall, there was an aversion for the location paired with ethanol $[F(1,30)=17.4]$. This effect of reinforcement did not significantly interact with the dose of ethanol $[F(2,30)=2.11]$, test cycle $[F(2,60)=1.9]$, or combination of these factors $[F(4,60)=2.11]$. However, an independent analysis of the third test alone revealed a statistically significant effect of dose $[F(2,30)=4.00]$. Subsequent polynomial comparisons revealed that the $2-\mathrm{g} / \mathrm{kg}$ dose produced a larger location aversion than either of the lower two doses $[F s(1,20)>4.81)]$, which did not significantly differ from one another $(F<1)$.

\section{Discussion}

The present experiment examined simultaneously the conditioned effects of ethanol on flavor and location cues. The results of this experiment were very clear. At all three doses examined, .5, 1.0, and $2.0 \mathrm{~g} / \mathrm{kg}$, ethanol conditioned an aversion for the location paired with the drug, the highest dose yielding the greatest aversion. The conditioned flavor effects were more complex: the high dose produced an aversion, the middle dose no clear effect, and the low dose yielded a preference. Thus, depending on the nature of the cue and dose paired with the drug, a preference, no effect, or an aversion was obtained.

The demonstration of a conditioned aversion to location cues paired with ethanol confirms the findings of Cunningham $(1979,1981)$, in which doses of $1.0,1.5$, and $2.0 \mathrm{~g} / \mathrm{kg}$, intraperitoneally (ip), all yielded an aversion to a location paired with ethanol. In his studies, rats were either water deprived or not. The present study extends these findings, demonstrating that a location aversion can also be obtained under conditions of food deprivation, and at lower doses.

Based on the results reported by Stewart and Grupp (1981), it was anticipated that using hungry rats and providing food in the context of the location conditioning paradigm would yield a preference for the location associated with ethanol. In their study, rats received an ip injection of ethanol $(.25, .5$, or $1.0 \mathrm{~g} / \mathrm{kg}$ for different groups) and were confined to one location; control injections preceded confinement in another location. For half the animals, training was conducted with food or without food present in both locations. In the conditions without food,

Table 1

Mean Percentage of Time Spent on the Side of the Shuttlebox Paired With Each Dose of Ethanol During Training for Each of the Three Tests During Experiment 1

\begin{tabular}{cccc}
\hline Dose* & Test 1 & Test 2 & Test 3 \\
\hline .5 & .43 & .43 & .44 \\
1.0 & .46 & .40 & .47 \\
2.0 & .48 & .36 & .30 \\
\hline
\end{tabular}

*In grams per kilogram. 
an aversion was conditioned at the highest dose but no statistically significant effects were observed with the lower doses. With food, however, a preference was conditioned at the middle dose, $.5 \mathrm{~g} / \mathrm{kg}$, but no effects were obtained at the other doses, suggesting that the aversion obtained with the highest dose was attenuated by the presence of food. In contrast to the findings of Stewart and Grupp, evidence for a conditioned location aversion was obtained here. Thus, the results of the present study did not confirm their demonstration of a conditioned location preference. However, the procedures of the present study differed from those of Stewart and Grupp along several dimensions. Most salient were: route of drug administration (ip in their study, and ig in the present study), duration of exposure to the location cues following ethanol administration (30 min in their study, and $20 \mathrm{~min}$ here), nature of food (Noyes pellets in their study, and sucrose here), and discriminative odor cues (present in their study, and not here). In any case, the results of the present experiment suggest that the motivational state of hunger and/or the consumption of food is not sufficient to account for the conditioning of a location preference found by Stewart and Grupp.

Of particular interest in the present results was the finding that the low dose, $.5 \mathrm{~g} / \mathrm{kg}$, of ethanol conditioned a preference for the flavor associated with it. This is of interest for two reasons. First, while a preference was conditioned to the flavor associated with ethanol, an aversion was simultaneously conditioned to the location cue. This confirms previous findings showing that a drug is not necessarily singular in its reinforcing properties (e.g., Sherman, Pickman et al., 1980).

Second, the demonstration of an ethanol-conditioned flavor preference is a novel finding. Among all the studies reporting flavor aversions conditioned with ethanol, Eckardt et al. (1974) used procedures most comparable to those employed here. They conducted conditioning with a nearly identical dose of ethanol $(.4 \mathrm{~g} / \mathrm{kg})$ and, as in the present study, they provided a calorically rich solution as a component of their flavor-conditioned stimulus. However, in contrast to the present study, they administered ethanol intraperitoneally rather than intragastrically. Lester et al. (1970) have shown that, with ethanol, intraperitoneal administrations are at least twice as effective as stomach intubation in yielding conditioned taste aversions. Consequently, the dose employed by Eckardt et al. would functionally be greater than the $.5-\mathrm{g} / \mathrm{kg}$ dose that yielded the conditioned flavor preference in the present study. Relevant to this point, higher doses in the present study produced no effect or conditioned a flavor aversion. Perhaps, in this way, route of administration accounted for the difference in results.
Another possibly important difference between their procedures and those employed here is that the animals in the present experiment were food deprived, whereas their animals were water deprived. Since ethanol is a calorically rich substance, it may be that the development of a conditioned flavor preference is based on its food value. Naturally, in animals that are not food deprived, the caloric effects of ethanol would not be as reinforcing as would be expected in hungry animals, and the aversive consequences of the drug would be more clearly manifested. This argument suggests that the conditioned flavor preference observed in the present study was due to the caloric contribution of ethanol, rather than to the possible rewarding effects of mild intoxication. Experiment 2 was undertaken to test this possibility.

\section{EXPERIMENT 2}

Several studies suggest that a neutral flavor paired with the postingestional consequences of a calorically rich substance can become preferred (Booth, Lovett, \& McSherry, 1972; Holman, 1975; LeMagnen, 1957a, 1957b). Thus, it is possible that the caloric contribution of the low dose of ethanol used in Experiment 1 was the reinforcing mechanism underlying the conditioning of a flavor preference. However, it should be noted that although the caloric contribution of the low dose of ethanol $(.5 \mathrm{~g} / \mathrm{kg})$ may account for the conditioned flavor preference, the fact that the higher dose $(2.0 \mathrm{~g} / \mathrm{kg})$, with the attendant increase in calories, conditioned a flavor aversion, suggests that at higher doses ethanol has aversive properties that outweigh any possible positive caloric effect.

The present study was undertaken (1) to assess the possibility that calories were sufficient to induce the conditioned flavor preference observed in Experiment 1 , and (2) to assess the nature of the conditioned effect when ethanol provides no caloric advantage over the control treatment. To address these issues, one group of rats was trained with the low dose of ethanol described in Experiment 1. A second group of rats received the same training, except that an isocaloric solution of glucose was used instead of ethanol. Lastly, for a third group of rats, one distinctive set of flavor-location cues was paired with the low dose of ethanol, whereas the opposite set of cues was paired with the isocaloric glucose solution, so that ethanol provided no caloric advantage.

\section{Method}

Subjects. The subjects were 36 male Sprague-Dawley rats obtained from Simonsen Labs in Gilroy, California. All other subject-related information is described in Experiment 1 . 
Apparatus and Drugs. The apparatus and drinking solutions were the same as those in Experiment 1. The solutions intubated were distilled water alone, distilled water mixed with ethanol to yield a $.5-\mathrm{g} / \mathrm{kg}$ dose or an isocaloric, $9.166 \%(\mathrm{w} / \mathrm{v})$ solution of glucose. The volume intubated was $10 \mathrm{ml} / \mathrm{kg}$.

Procedure. As in Experiment 1, all rats were sham intubated and preexposed to a $5 \%$ sucrose solution prior to their first training day. Subsequently, rats were randomly assigned to one of three groups $(n=12)$. The drug schedule during training varied for each of the three groups. Group $\mathrm{H}_{2} \mathrm{O}$-ETOH received water on Mondays and Thursdays and ethanol on Tuesdays and Fridays. Group $\mathrm{H}_{2} \mathrm{O}$-Glucose received water on Mondays and Thursdays and glucose on Tuesdays and Fridays. Lastly, Group Glucose-ETOH received glucose on Mondays and Thursdays and ethanol on Tuesdays and Fridays. Training was conducted for 6 weeks, until each group had had 12 pairings of each intubated drug with a distinctive set of location and flavor cues. Testing of flavor and location preferences was conducted on the Sunday following the last drug presentation. All further procedural details followed those described in Experiment 1 . During training, two rats died, one from Group $\mathrm{H}_{2} \mathrm{O}$-Glucose and one from Group $\mathrm{H}_{2} \mathrm{O}-\mathrm{ETOH}$.

All statistical tests were conducted with the ANOVA described in Experiment 1. However, the reinforcing drug now arbitrarily refers to the substance presented on Tuesdays and Fridays. Missing data were analyzed by the method suggested by Hicks (1964).

\section{Results}

Figure 2 presents the mean amount of solution consumed during training and testing for all three groups. During training, rats in Group $\mathrm{H}_{2} \mathrm{O}-\mathrm{ETOH}$ (see top panel of figure) consumed significantly more of the solution paired with ethanol than that paired with water $[F(1,9)=10.7]$, and this effect increased over trials $[F(11,99)=7.8]$. Consistent with these training results, the flavor test revealed a statistically significant preference for the ethanol-paired solution $[F(1,9)=22.8]$. These data replicate the results of Experiment 1 showing a conditioned preference for a flavor paired with a low dose $(.5 \mathrm{~g} / \mathrm{kg})$ of ethanol.

During training, the rats of Group $\mathrm{H}_{2} \mathrm{O}$-Glucose drank significantly more of the glucose-paired solution than of the water-paired solution $[F(1,9)=13.0]$, and the magnitude of this effect generally increased over training sessions (see middle panel). A reversal of this trend occurred on Trial Pair 8, contributing to the significant trials $\times$ reinforcer interaction $[F(11,99)=3.33]$. It is not clear what caused this minor perturbation in the data. Consistent with the overall trend, the test results show a clear conditioned preference for the flavor associated with glucose $[F(1,9)=19.9]$. A statistical comparison of the reinforcement effect observed in Groups $\mathrm{H}_{2} \mathrm{O}-\mathrm{ETOH}$ and $\mathrm{H}_{2} \mathrm{O}$-Glucose failed to reveal a significant groups $\times$ reinforcement interaction $(F<1)$, suggesting that the magnitude of the conditioned preference obtained with ethanol and an isocaloric, glucose solution were highly comparable. Thus, it appears that the caloric contribution of ethanol may be sufficient to condition a flavor preference.

The rats of Group Glucose-ETOH failed to show a statistically significant difference in consumption during training $[F(1,10)=4.12]$, although greater consumption of the glucose-paired solution was suggested (see bottom panel). However, the more sensitive two-bottle preference test (Grote \& Brown, 1971) revealed a statistically significant preference for the glucose-paired solution over the ethanolpaired solution $[F(1,10)=15.6]$. This result suggests that when ethanol provides no caloric advantage, either it does not condition a flavor preference or an aversion is actually conditioned.

The results of the location test failed to yield a significant preference for either side of the shuttlebox for any of the three groups [all $\mathrm{Fs}(1,10)<2.0$ ]. The absence of a conditioned location aversion in Group $\mathrm{H}_{2} \mathrm{O}$-ETOH fails to replicate the conditioned location aversion obtained with this dose $(.5 \mathrm{~g} / \mathrm{kg})$ in Experiment 1. These data question the reliability of the conditioned location aversion obtained with this dose. It is clear, however, that conditioning with flavor cues provides a more reliable index of the conditioned effects of ethanol at this low dose.

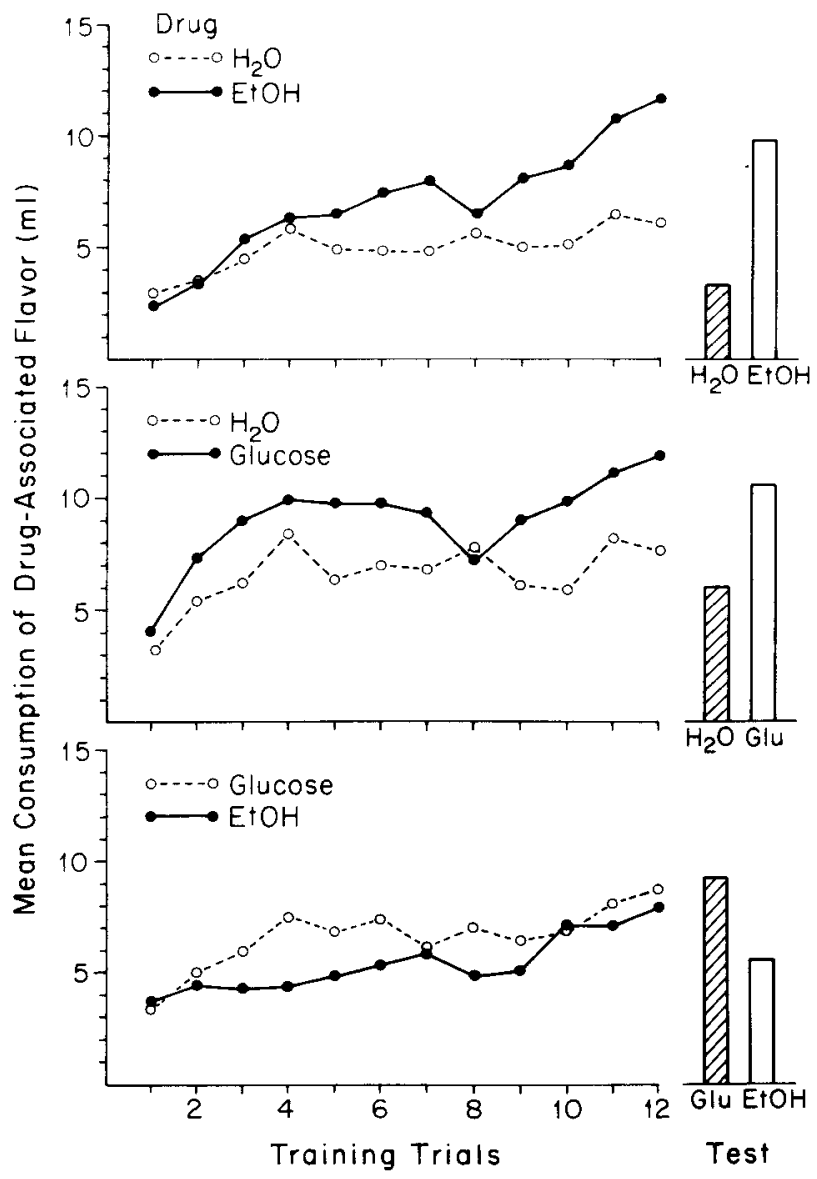

Figure 2. Mean consumption of flavors associated with ethanol (ETOH), isocaloric glucose (Glu), or water $\left(\mathrm{H}_{2} \mathrm{O}\right)$ during training and testing. 


\section{Discussion}

The results of Experiment 2 suggest that the conditioned preference for a flavor associated with a low dose of ethanol may be attributed to ethanol's caloric content rather than any unique effect of the drug. This interpretation is supported by the finding that an isocaloric solution of glucose yielded a conditioned flavor preference of comparable magnitude. Although it is possible that different mechanisms account for the flavor preferences conditioned with ethanol or glucose, the results of Experiment 2 suggest that caloric restoration may be sufficient to explain the flavor preference conditioned with ethanol.

The fact that the highest dose of ethanol used in Experiment $1(2.0 \mathrm{~g} / \mathrm{kg})$ conditioned a flavor aversion indicates that higher doses produce aversive consequences that outweigh the benefits of caloric restoration. That rats were found to prefer an isocaloric, glucose-paired flavor to an ethanol-paired flavor (Group Glucose-ETOH) suggests that when the caloric advantage of ethanol is canceled even a low dose of ethanol may be argued to have aversive properties.

Although we have suggested that caloric restoration served as the reinforcer in conditioning a flavor preference for the $.5-\mathrm{g} / \mathrm{kg}$ doses of both ethanol and glucose, other mechanisms are possible. Bolles, Hayward, and Crandall (1981) have reported evidence showing that oral cues correlated with caloric benefit, such as the cues provided by starch, may serve as the reinforcer rather than the caloric benefit per se. It is possible that oral cues provided by ethanol and glucose were present in the experiments presented here as well. Although caution was taken to rinse the tube used in gastrically presenting ethanol or glucose prior to intubation, it is possible that a weak solution remained on the tube when it passed over the tongue. Also, after the contents of the tube were injected into the stomach, it is possible that the external side of the tube was bathed in the substance and subsequent withdrawal of the tube provided such oral stimulation. Thus, taste cues, rather than caloric restoration, may have reinforced the conditioned flavor preference. The present study does not discriminate between these two possibilities.

In conclusion, these data show that ethanol readily conditions an aversion to both flavor and location cues at relatively high doses and may be shown to condition a preference for flavor cues at a low dose. It appears that the conditioned flavor preference obtained with the low dose of ethanol is a consequence of its caloric content rather than the intoxicating effects of the drug. It is not clear to what extent the nutritional value of ethanol con- tributes to the development of a preference for ethanol in humans. Interestingly, it is evident that social drinking accompanies meals. The results obtained here suggest that the initially satisfying effects of ethanol may be related, in part, to the rich caloric restoration provided by the drug.

\section{REFERENCES}

BEACH, H. D. Morphine addiction in rats. Canadian Journal of Psychology, 1957, 11, 104-112.

Bolles, R. C., Hayward, L., \& Crandall, C. Conditioned taste preferences based on caloric density. Journal of Experimental Psychology: Animal Behavior Processes, 1981, 7, 59-69.

Booth, D. A., Lovetr, D., \& McSherry, G. M. Postingestive modulation of the sweetness preference gradient in the rat. Journal of Comparative and Physiological Psychology, 1972, 78, 485-512.

Cappell, H., LeBlanc, A. E., \& Endrenyi, L. Aversive conditioning by psychoactive drugs: Effects of morphine, alcohol, and chlordiazepoxide. Psychopharmacologia, 1973, 29, 239-246.

Cunningham, C. L. Flavor and location aversions produced by ethanol. Behavioral and Neural Biology, 1979, 27, 362-367.

Cunningham, C. L. Spatial aversion conditioning with ethanol. Pharmacology, Biochemistry and Behavior, 1981, 14, 263-264.

Eck^rdt, M. J., Skurdal, A. J., \& Brown, J. S. Conditioned taste aversion produced by low doses of alcohol. Physiological Psychology, 1974, 2, 89-92.

Grote, F. W., \& Brown, R. T. Conditioned taste aversions: Two-stimulus tests are more sensitive than one-stimulus tests. Behavior Research Methods \& Instrumentation, 1971, 3, 311312.

Hicks, C. R. Fundamental concepts in the design of experiments. New York: Holt, Rinehart \& Winston, 1964.

Holman, E. W. Immediate and delayed reinforcers for flavor preferences in rats. Learning and Motivation, 1975, 6, 91-100.

LeMagnen, J. Effet de la durée du jeune post prandial sur l'establissement des appétits chez le rat blanc. Comptes Rendus de la Société de Biologie, 1957, 151, 229-231. (a)

LeMagnen, J. Etude d'un facteur post ingestif de l'establissement des appetits chez le rat blanc. Archives des Sciences Physiologiques, 1957, 11, 237-254. (b)

Lester, D. M., Nachman, M., \& LeMagnen, J. Aversive conditioning by ethanol in the rat. Quarterly Journal of Studies on Alcohol, $1970,31,578-586$.

Reicher, M. A., \& Holman, E. W. Location preference and flavor aversion reinforced by amphetamine in rats. Animal Learning \& Behavior, 1977, 5, 343-346.

Rossi, N. A., \& REID, L. D. Affective states associated with morphine injections. Physiological Psychology, 1976, 4, 269274.

Sherman, J. E., Pickman, C., Rice, A., Liegeskind, J. C., \& Holman, E. W. Rewarding and aversive effects of morphine: Temporal and pharmacological properties. Pharmacology, Biochemistry \& Behavior, 1980, 13, 501-505.

Sherman, J. E., Roberts, T., Roskam, S. E., \& Holman, E. W. Temporal properties of the rewarding and aversive effects of amphetamine in rats. Pharmacology, Biochemistry and Behavior, 1980, 13, 597-598.

Stewart, R. B., \& GRUPP, L. A. An investigation of the interaction between the reinforcing properties of food and ethanol using the place preference paradigm. Progress in Neuro-Psychopharmacology, 1981, 5, 609-613.

(Manuscript received July 27, 1982; accepted for publication September 20, 1982.) 